\title{
Information from dynamic length changes improves reliability of static ultrasound fascicle length measurements
}

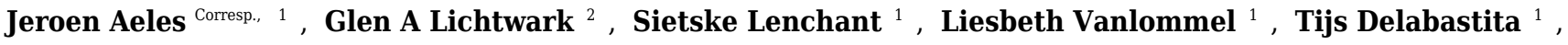 \\ Benedicte Vanwanseele ${ }^{1}$ \\ 1 Department of Kinesiology, KU Leuven, Leuven, Belgium \\ 2 School of Human Movement Studies, University of Queensland, Brisbane, Australia \\ Corresponding Author: Jeroen Aeles \\ Email address: jeroen.aeles@kuleuven.be
}

Purpose. Various strategies for improving reliability of fascicle identification on ultrasound images are used in practice, yet these strategies are untested for effectiveness. Studies suggest that the largest part of differences between fascicle lengths on one image are attributed to the error on the initial image. In this study, we compared reliability results between different strategies.

Methods. Static single-image recordings and image sequence recordings during passive ankle rotations of the medial gastrocnemius were collected. Images were tracked by three different raters. We compared results from uninformed fascicle identification (UFI) and results with information from dynamic length changes, or data-informed tracking (DIT). A second test compared tracking of image sequences of either fascicle shortening (initial-long condition) or fascicle lengthening (initial-short condition).

Results. Intra-class correlations (ICC) were higher for the DIT compared to the UFI, yet yielded similar standard error of measurement (SEM) values. Between the initial-long and initial-short conditions, similar ICC values, coefficients of multiple determination, mean squared errors, offset-corrected mean squared errors and fascicle length change values were found for the DIT, yet with higher SEM values and greater absolute fascicle length differences between raters on the first image in the initial-long condition and on the final image in the initial-short condition.

Conclusions. DIT improves reliability of fascicle length measurements, without lower SEM values. Fascicle length on the initial image has no effect on subsequent tracking results. Fascicles on ultrasound images should be identified by a single rater and care should be taken when comparing absolute fascicle lengths between studies. 
1 Information from dynamic length changes improves reliability of static ultrasound fascicle length measurements

3

4

$\frac{6}{7}$

8

9

10

11

12

13

14

16

17

18

19

20

21

22

23

24

25

26

27

28

29

30
Jeroen Aeles ${ }^{1}$, Glen A. Lichtwark ${ }^{2}$, Sietske Lenchant ${ }^{1}$, Liesbeth Vanlommel ${ }^{1}$, Tijs Delabastita ${ }^{1}$, Benedicte Vanwanseele ${ }^{1}$

${ }^{1}$ KU Leuven - University of Leuven Department of Kinesiology, Department of Kinesiology, KU Leuven, Leuven, Belgium

${ }^{2}$ University of Queensland - St. Lucia School of Human Movement Studies, University of Queensland, Brisbane, Australia

ORCID, Jeroen Aeles: 0000-0003-1514-4958

Corresponding author:

Jeroen Aeles

Department of Kinesiology

KULeuven

Leuven

Belgium

Jeroen.aeles@kuleuven.be

Other authors:

Glen Lichtwark

School of Human Movement and Nutrition Sciences

University of Queensland

Brisbane

Australia 
Abbreviations strategies.

Purpose. Various strategies for improving reliability of fascicle identification on ultrasound images are used in practice, yet these strategies are untested for effectiveness. Studies suggest that the largest part of differences between fascicle lengths on one image are attributed to the error on the initial image. In this study, we compared reliability results between different

Methods. Static single-image recordings and image sequence recordings during passive ankle rotations of the medial gastrocnemius were collected. Images were tracked by three different raters. We compared results from uninformed fascicle identification (UFI) and results with information from dynamic length changes, or data-informed tracking (DIT). A second test compared tracking of image sequences of either fascicle shortening (initial-long condition) or fascicle lengthening (initial-short condition).

Results. Intra-class correlations (ICC) were higher for the DIT compared to the UFI, yet yielded similar standard error of measurement (SEM) values. Between the initial-long and initial-short conditions, similar ICC values, coefficients of multiple determination, mean squared errors, offset-corrected mean squared errors and fascicle length change values were found for the DIT, yet with higher SEM values and greater absolute fascicle length differences between raters on the first image in the initial-long condition and on the final image in the initial-short condition.

Conclusions. DIT improves reliability of fascicle length measurements, without lower SEM values. Fascicle length on the initial image has no effect on subsequent tracking results. Fascicles on ultrasound images should be identified by a single rater and care should be taken when comparing absolute fascicle lengths between studies.

\begin{tabular}{ll}
\hline CMD & Coefficients of multiple determination \\
DIT & Data-informed tracking \\
ICC & Intra-class correlations \\
MSE & Mean squared error \\
SEM & Standard errors of measurement \\
UFI & Uninformed fascicle identification \\
\hline
\end{tabular}




\section{Introduction}

It has long been established that muscle fascicle length changes are decoupled from the length changes of the entire muscle-tendon unit (Hoffer et al. 1989; Fukunaga et al. 2001). Because of this, inferences about fascicle behavior from kinematic data is difficult and can lead to errors, especially in muscle-tendon units with relatively long tendons. Objective data of muscle fascicle lengths is therefore needed. As such, an increased interest in identifying muscle fascicle geometry has been emerging, as muscle fascicle length and orientation can provide valuable information about muscle performance (e.g. Abe et al. 2000; Blazevich 2006; Farris et al. 2016) as well as clinical or training adaptations (Mohagheghi et al. 2007; Blazevich et al. 2014; Hoffman et al. 2016). B-mode ultrasound imaging is the most commonly used method to determine muscle fascicle geometry, because it is cost- and time-effective and it allows for measurements during dynamic tasks, such as walking (Fukunaga et al. 2001; Ishikawa et al. 2005), running (Lichtwark and Wilson 2006; Ishikawa et al. 2007), and jumping (Kurokawa et al. 2001; Farris et al. 2016).

Acquired ultrasound images are often analyzed by manually identifying muscle fascicles to obtain information about fascicle length and orientation (Cronin et al. 2011; Cronin and Lichtwark 2013). This has the potential to result in low reliability and low repeatability because of the subjective nature of this analysis. Studies on the reliability of fascicle identification from ultrasound images during a wide range of tasks, including measurements of muscle in a relaxed and contracted state, during walking, running and jumping, have reported standard error of measurement (SEM) percentages of $4.3-14.2 \%$ for inter-session (Kwah et al. 2013), $0.0-8.3 \%$ for inter-image (Kwah et al. 2013) and 3.8 - 7.5\% for inter-rater (König et al. 2014; McMahon et al. 2016) analyses. Overall, these values remain rather high, considering the effect sizes generally reported in cross-sectional or longitudinal training studies (10-19\%) (Abe et al. 2000; Fukutani and Kurihara 2015; Timmins et al. 2016) and in studies that compare fascicle length changes between various conditions within similar dynamic tasks (9-14\%) (Lichtwark and Wilson 2006; Farris and Sawicki 2012; Brennan et al. 2016). It is therefore essential to explore methods for lowering fascicle identification errors and increasing reliability.

Even though manual fascicle identification remains the "gold standard", it is likely to induce subjective errors, for example due to experimenter bias (Cronin et al. 2011). Various efforts have been made in the last two decades to objectify fascicle tracking on image sequences by development of (semi-) automated image-processing algorithms. These algorithms are mainly based on cross-correlation methods (Loram et al. 2003; Herbert et al. 2011) or optical flow methods (Magnusson et al. 2003, Rana et al. 2009, Cronin et al. 2011; Farris and Lichtwark 2016) and allow for an automated tracking of visible structures on ultrasound image sequences. Most of these proposed automated methods have proven to accurately match manual trackings and allow for a more objective analysis. This makes automated processing an appealing alternative for manual processing as it is also less time-consuming and thus more efficient. In 
97 98

spite of these advantages of automated tracking, the errors made between consecutive trackings still remain rather high. Gillet et al. (2013) reported standard errors between 5 and $10 \%$ of absolute fascicle length, similar to manual tracking (Gillet et al. 2013; Kwah et al. 2013). They stated that this is most likely contributed to by the errors made on the required initial manual input, which is often required on the first frame of an image sequence for this type of automated tracking algorithm (Herbert et al. 2011; Cronin et al. 2011; Gillet et al. 2013; Farris and Lichtwark 2016). These authors suggest that the initial length estimate variability likely explains the greatest part of variability in fascicle tracking reliability and recommend efforts for improvement of the initial fascicle tracking.

Many studies on static muscle fascicle architecture use single-image ultrasound recordings (Abe et al. 2000; Karamanidis et al. 2011; Franchi et al. 2014; Aeles et al. 2017). For these studies it may be of even greater importance to find methods for improving the fascicle identification reliability, as the absolute fascicle lengths are generally the main outcome. A natural consequence of this single-image method is that there is no information prior to or after the initial image to help guide the researcher for accurate and reliable fascicle identification. It is often believed by researchers in this particular field that tracking of image sequences is more reliable than fascicle identification on single images as the researcher can focus on movement patterns to identify the correct fascicle orientation on the image sequences. Despite the recommendations by Gillet et al. in 2013, no studies, to the best of our knowledge, have focused on improving fascicle identification reliability for single-image and image sequence ultrasound recordings.

The aim of this study was therefore, to compare different strategies for ultrasound fascicle length measurements, commonly used in practice, in order to increase fascicle identification reliability between different raters. We used images of the medial gastrocnemius as this is a popular muscle for ultrasound measurements in vivo because it is a superficial muscle with relatively short muscle fascicles and it has important contributions during tasks such as walking, running and jumping (Fukunaga et al., 1997; Lichtwark and Wilson, 2007). Our first strategy test compared images from single-image fascicle identification, with no information before and after the image, i.e. uninformed fascicle identification (UFI) and the first images of image sequences with fascicle information after the initial image, i.e. data-informed tracking (DIT). We hypothesized the UFI to yield larger between-rater differences and lower reliability compared to the DIT. For our second strategy test, we compared ultrasound image sequences with two different fascicle starting lengths. We hypothesized lower between-rater differences and greater reliability when tracking fascicle shortening (initial frame has long fascicle lengths; further named 'initial-long condition') compared to tracking fascicle lengthening (initial frame has short fascicle lengths; further named 'initial-short condition'). This hypothesis was based on the premise that fascicles are more clearly visible at long lengths, when they have their lowest pennation angle and 
136 therefore the fascicle structure is more perpendicular to the ultrasound waves, creating more

137 defined images (Lichtwark 2017).

138

139

140

141

142

143

144

145

146

147

148

149

150

151

152

153

154

155

156

157

158

159

160

161

162

163

164

165

166

167

168

169

170

171

172

\section{Materials \& Methods}

\section{Participants}

Ultrasound image sequences of passive ankle joint rotations were collected for 28 participants (13 female, 15 male; body height $=179.73 \pm 8.21 \mathrm{~cm}$; body mass $=73.01 \pm 9.21 \mathrm{~kg})$. From these 28 participants, 5 were randomly selected and single-image ultrasound recordings were taken for an intra-rater reliability test and the UFI analyses. For the DIT comparisons, all 28 participants were included. An overview of data collection is shown in figure 1. All participants confirmed to participate in the study by written informed consent. Three independent researchers, who will be referred to as 'raters' were asked to participate in the study for the fascicle identification of the ultrasound images. All raters were experienced with identification and tracking of medial gastrocnemius fascicles and were not informed about the purpose of the study. The study was approved by the local ethics committee (ethische commissie onderzoek UZ / KU Leuven; approval number - S57477 - ML11371) and conforms to the recommendations of the Declaration of Helsinki.

\section{Experimental protocol}

For the assessment of the intra-rater reliability and the UFI analysis, single-image recordings of the medial gastrocnemius fascicles of the left and right leg were collected for 5 subjects. For each leg and subject, three images were taken at approximately the same mid-image location of the muscle with the ankle joint in a maximal dorsiflexed position. The fascicle length at this joint angle is equal to the fascicle length used in the initial-long condition for the DIT and thus these images were used to compare the UFI and DIT strategies. For the DIT and image sequence tracking analyses, ultrasound images of the medial gastrocnemius fascicles of the left and right leg were collected during passive rotations of the ankle joint. Subjects were laying in prone position on a table, with the knee and hip joint fully extended. During this passive trial, the ankle joint was manually rotated three times over the full range of motion with the subject fully relaxed. All ultrasound image recordings were captured using a Telemed Echoblaster 128 CEXT system (UAB Telemed, Vilnius, Lithuania). B-mode images were collected at $30 \mathrm{~Hz}$ for all measurements using a 60mm, 128-element linear transducer (UAB Telemed, Vilnius, Lithuania, LV 7.5/60/128Z-2). The transducer was positioned longitudinally over the mid-belly of the medial gastrocnemius for all measurements. During imaging, the transducer was held at the same location by the operator. Minimal movement of the transducer during the ankle joint rotations was confirmed with markers on the transducer and on the knee joint using a motion capture system (Vicon, Oxford Metrics, UK). 
173 Image processing protocol

174 All ultrasound images were processed using fascicle identification software (Farris and 175 Lichtwark 2016) in MATLAB R2014 (The Mathworks, Natick, US). For this study, all raters

176 were asked to identify the fascicle end-points manually on each frame. All raters used the same 177 fascicle identification techniques: at first, two lines were manually drawn on the image, one over 178 the deep aponeurosis and one parallel to the muscle fascicles with attachments to the deep and 179 superficial aponeurosis. The length of this line represented the fascicle length and was calculated 180 based on its relative length to the image depth, which was set during the measurements at 50 $181 \mathrm{~mm}$. Each rater received the same instructions for the fascicle identification: they were asked to 182 identify the initial image of each image sequence first and then, for the image sequences, manually track the fascicle by adjusting the fascicle end-points on each frame. For the image sequences, the raters were allowed to watch the sequences prior to fascicle identification and to play the sequences back and forth during the tracking. Raters could adjust the fascicle endpoints on the initial image and on all subsequent images based on the fascicle information they obtained from the dynamic length changes of the fascicle on the subsequent frames (i.e. DIT). For all images, raters were instructed to focus on the middle region of the image for identification of the fascicle, as fascicle behavior may differ throughout the muscle belly (Lichtwark et al. 2007) and this ensures that most of the fascicle is visible in the image. For the fascicles with attachments outside of the image, visual linear extrapolation of the superficial aponeurosis only was used by the raters, ensuring that at least the attachment on the deep aponeurosis was visible throughout the whole image sequence. For the tracking of the passive rotation image sequences, the second of three full rotation cycles was extracted and split at the maximum fascicle length, resulting in one image sequence file with fascicle shortening (= initiallong) and one with fascicle elongation (= initial-short).

All raters followed the same order of analyzing the different ultrasound recordings. They were instructed to first track the static ultrasound images used for the reliability test and UFI analysis. Afterwards, each rater tracked the image sequences of the passive rotations. All raters first tracked all files containing the initial-long images and then all files containing the initial-short. Due to technical issues (e.g. no data of full ankle joint range of motion or missing data from one of the raters), 3 files from the initial-long condition and 6 files from the initial-short condition were excluded. As such, 53 sets of initial-long and 50 sets of initial-short image sequences were used for further analyses. In order to exclude tracking biases, all data was randomized per set of recordings and blinded for the raters. After data processing, fascicle length results from the left and right leg were combined for all data sets for analyses.

\section{Data analyses}

209 For the comparison between UFI and DIT, inter-rater intra-class correlations (ICC) $(2,1$; single) and SEM values were calculated between the different raters using SPSS v.22 software (IBM SPSS, New York, U.S.A.). This was done using all of the 30 single-image recordings for the UFI 
212 condition and the first image of each of the 53 initial-long image sequences for the DIT

213 condition.

214

215

216

217

218

219

220

221

222

223

224

225

226

227

228

229

230

231

232

233

234

235

236

237

238

239

240

241

242

243

244

245

246

247

248

249

250
Each of the analyses further described were done for both initial-long and initial-short fascicle conditions. All image sequence waveforms were low-pass filtered using a fourth order Butterworth filter (MATLAB R2014, The Mathworks, Natick, US). The initial-long and initialshort strategies were compared for the DIT condition, using the first image of each of the 53 initial-long and the first image of each of the 50 initial-short image sequences. Coefficients of multiple determination (CMD) were calculated between the waveforms of two of the raters and the corresponding waveform of a reference rater. The reference rater was chosen based on the results of an intra-rater ICC (2,1; single) and SEM analysis. For this, 10 of the 30 single-image recordings that were used for the UFI, were analysed three times non-consecutively by each rater for the assessment of the intra-rater reliability. ICC values for all raters were good to excellent with the lowest ICC equal to 0.78. SEM values were good to excellent with a maximal value of $3.73 \mathrm{~mm}$. The ICC value of the reference rater was very high (0.98) and SEM was low (0.81 $\mathrm{mm})$. Calculating the CMD values between the reference rater and each of the other raters resulted in two CMD values per set, which were first averaged per set and then averaged over all sets. To assess the absolute error, the mean squared error (MSE) was calculated. MSE was calculated as the mean of the squared difference between the waveforms of each of the rater at every data point. Again, MSE values were first averaged per set and then averaged over all sets. To test for the influence of the variability between raters on the initial image, we used the methods described by Gillet et al. (2013): all waveforms were corrected for their respective initial fascicle length and MSE analyses was repeated (= offset-corrected condition) (fig. 2B). This was done by subtracting the fascicle length of the first image from each data point of the respective waveforms.

To evaluate the practical relevance and impact of the different strategies, the differences between raters for physiologically-relevant parameters were calculated (fig. 2A). A first parameter was the total fascicle length change over the full ankle joint range of motion, which was calculated as the difference between the longest and shortest length of the fascicle on one waveform. Secondly, to test for outcome differences in static muscle architecture studies that use singleimage recordings, absolute fascicle lengths were compared for the three raters on the first and last image of each waveform. Results were calculated both as absolute and relative differences between the two respective raters. The relative differences were calculated as the percentage of the average absolute fascicle length between the two respective raters. For all these parameters, the difference between the three raters were calculated per set of waveforms, after which these differences were first averaged per set of waveforms and then over all sets.

\section{Results}


251 ICC values were greater for the DIT as compared to the UFI, however similar SEM values were 252 found (table 1). Between the initial-long and initial-short conditions, similar ICC values were

253 found for the DIT. However, a higher SEM value was found in the initial-long condition

254

255

256

257

258

259

260

261

262

263

264

265

266

267

268

269

270

271

272

273

274

275

276

277

278

279

280

281

282

283

284

285

286

287

288

289

compared to the initial-short condition.

CMD values were very high for both the initial-long and initial-short conditions and not significantly different between both conditions (table 2). MSE values were not significantly different between the two conditions for both the original waveforms as well as the offsetcorrected waveforms. However, MSE significantly decreased after offset correction $(\mathrm{p}<0.001)$ in both conditions.

Differences in total fascicle length change between the reference rater and raters 1 and 2 are shown in figure 3. Neither absolute nor relative differences between raters were significantly different between the initial-long and initial-short conditions (table $3+4$ ). Absolute differences in fascicle length between the different raters were greater on the initial image in the initial-long condition compared to the initial-short condition $(\mathrm{p}=0.005)$. This difference was non-existent when comparing the relative differences. On the final image, the absolute differences in fascicle length were smaller in the initial-long condition compared to the initial-short condition $(\mathrm{p}=$ 0.003). Again, this was not found when comparing the relative differences.

\section{Discussion}

In this study, we tested different strategies for ultrasound fascicle length measurements that are commonly being used in practice. Our first aim was to compare two strategies for use in studies that make use of single-image recordings for fascicle length. We made a comparison between single-image recordings with no information before or after the image of interest (UFI) and the initial frame of image sequences with information after the image of interest (DIT). There was a substantial difference between the DIT and UFI in ICC scores. However, SEM values were not different between the two conditions, suggesting that although the identification error was not influenced by the condition, the error was made in a more consistent manner. Raters often rely on the movement of the fascicle and changes in its orientation and length to help identify the correct movement patterns during fascicle identification. As this cannot be done on single-image fascicle lengths, our results suggest that it is worthwhile to record image sequences with fascicle movement, even for studies interested in single-image analyses. SEM values between different raters remain rather high (4.15 and $4.29 \mathrm{~mm}$ for DIT and UFI respectively) for both strategies, especially compared to intra-rater SEM values (average of $2.59 \pm 1.56 \mathrm{~mm}$ ), but are within the range reported in other studies (König et al. 2014; McMahon et al. 2016).

As the DIT strategy proved to be a more reliable method for single-image outcomes and it can be used in studies interested in fascicle length changes during dynamic activities, we aimed at 
290 further improving this method by employing our second strategy. For this strategy we compared

291

292

293

294

295

296

297

298

299

300

301

302

303

304

305

306

307

308

309

310

311

312

313

314

315

316

317

318

319

320

321

322

323

324

325

326

327

328

329

two conditions with different fascicle lengths on the initial image, either initial-long or initialshort. ICC values for both conditions were similar, yet, against expectations, the SEM value for the initial-long condition was almost $1 \mathrm{~mm}$ greater compared to the initial-short condition. A reasonable explanation for this is that many times, in the initial-long images, fascicle attachments to the superficial aponeurosis are outside of the visible image area. In these cases, linear extrapolation of both the tracked fascicle and the aponeurosis was used visually by the raters, a common method in fascicle length measurements for ultrasound images. As such, efforts should be made to avoid this type of error, for example by using suggested methods in which only the visible part of the fascicle is identified and then the whole fascicle length is calculated using extrapolation in the analyses only after identification of the fascicle on the image (Finni et al. 2003; Seiberl et al. 2010). However, even though this method is very useful in muscles with minimal fascicle curvature such as the medial gastrocnemius, they should be used with care in other muscles such as the biceps femoris, which shows fascicles with substantial curvature (as shown on figure 1a in Seymore et al., 2017). To verify that this greater difference in fascicle length between the raters was actually due to the longer fascicle length together with the associated difficulties that were addressed earlier, and not due to the fact that this was the initial frame of the image, we compared the absolute MSE values between the raters on both the initial and final image of each waveform. Here we again found a significant difference between the two conditions, with a larger difference between the raters in the initial-long compared to the initialshort condition on the first image but the opposite, a larger difference between the raters in the initial-short compared to the initial-long conditions, on the final image. As such, it appears that this difference is indeed mainly a result of the difference in absolute fascicle length at that respective image, as also suggested by Gillet et al. (2013). Indeed, our results clearly show that the greater difference between raters on images with long fascicle length is due to the greater fascicle length and is independent of its relative position (i.e. first or final image) in the waveform, as the differences relative to the average fascicle length on that image were equal between the initial-long and initial-short condition for either the first $(8.98 \pm 5.12 \%$ versus 8.12 $\pm 4.29 \%$ respectively) or last $(8.34 \pm 4.97 \%$ versus $8.89 \pm 4.55 \%$ respectively) frame.

Since many movements commonly assessed with ultrasound are cyclical movements, such as gait or passive and active ankle joint rotations, researchers are often allowed to choose at which part of an image sequence cycle they would like to start their fascicle tracking. Through personal communication, we established that many researchers in practice prefer to start tracking on either shortened fascicles, preferring the initial-short strategy or lengthened fascicles, preferring the initial-long strategy as it is commonly believed that this results in lower fascicle identification errors for the rest of the image sequence. The longer fascicle in the initial-long strategy in pennate muscles is generally accompanied by a lower pennation angle, decreasing the angle of incidence of the sound waves sent out by the transducer and allowing more reflections of the sound waves. This generally increases the visibility of the imaged structures (Lichtwark, 2017). 
330 However, our results indicate no difference in inter-rater reliability, inter-rater waveform

331

332

333

334

335

336

337

338

339

340

341

342

343

344

345

346

347

348

349

350

351

352

353

354

355

356

357

358

359

360

361

362

363

364

365

366

367

368

369 similarity (CMD), absolute differences between raters (MSE), and total fascicle length change differences between the two conditions. Overall, these results suggest that the length of the fascicle on the initial image does not influence the reliability of the subsequent tracking in image sequences. However, it must be noted that the image sequences used in this study were not cyclical, as only one joint rotation was used for analysis and cut at the maximum fascicle length to obtain the initial-short and initial-long image sequences. Image sequences of cyclical motions that have multiple waves introduce the advantage of the fascicle returning to a similar length, allowing the rater to compare the fascicle lengths at similar sections of the wave and adjusting the fascicle identification accordingly.

The conclusions in this study were drawn from our analyses of the medial gastrocnemius muscle fascicles. However, other muscle-tendon units often present different fascicle geometries, for example the knee extensors have much longer fascicles and more curvature of the fascicles (Seymore et al., 2017). As the strategies discussed in this paper have not been tested in these muscle-tendon units, we should be careful in generalizing these results. For example, due to the longer fascicles in the vastus lateralis, it is likely that large portions of the fascicle lies outside the image when using short transducers (e.g. the $60 \mathrm{~mm}$ transducer used in this study), especially when stretching the fascicles to long lengths. As such, in these muscle-tendon units the effect between the initial-short and initial-long condition may be more significant than shown by the results in the current study.

When we corrected the fascicle length on each frame for the fascicle length on the initial image, MSE was significantly lowered. This shows that a large part of the variability between fascicle lengths of different raters is explained by the variability in fascicle length on the initial image. This finding was also reported by Gillet et al. (2013) when comparing automated trackings with different initial-image inputs by different raters. Combined with the very high CMD values found in this study, we can conclude that there was high similarity between the tracking of waveforms by different raters and that the main difference arises in the initial fascicle length estimation. As such, relative length changes of the fascicle are highly reliable between different raters, but less so in terms of absolute values.

Even though offset-correction is a successful strategy for lowering MSE values between different raters, it has no effect on physiologically-relevant parameters such as the total length change of the fascicle. To our knowledge, no other study has tested the reliability between raters for analyses of total fascicle length changes on ultrasound images. Yet, the observed differences in these length changes between raters in this study are in close proximity to reported effect sizes in total fascicle length changes (4-6 mm) of other studies (Duclay et al. 2009; Sakuma et al. 2012; Theis et al. 2013; Blazevich et al. 2014). Indeed, as can be seen on figure 3, these effect sizes are well within the range of the coefficients of repeatability (calculated as 1.96 times the standard 
370

371

372

373

374

375

376

377

378

379

380

381

382

383

384

385

386

387

388

389

390

391

392

393

394

395

396

397

398

399

400

401

402

403

404

405

406

407

408

409

deviation of differences between two raters). This means that there is a $95 \%$ probability that differences in total fascicle length change between raters are greater than the actual effect size. As such, we suggest that processing of image sequences in studies that are interested in parameters such as absolute fascicle length changes, should not be performed by different raters, as could sometimes be preferable in large studies. Furthermore, this means that care should be taken when comparing absolute values of fascicle length changes between different studies. However, as we did not test within-rater reliability on the image sequences, we cannot conclude that processing of the images by a single rater is more reliable. Yet, it seems that the differences between the raters are rather consistent (fig. 3) in terms of over- or underestimating the total fascicle length change compared to the other raters. Together with the high CMD values between the waveforms of each set, this suggests that raters are consistent in their tracking both within one image sequence as well as between the different image sequences.

In this study we have only performed analyses on static or passive ultrasound images. During active movements, the fascicle pennation angle can be higher compared to our initial-short condition, potentially causing reduced image quality. Yet, as we did not find any differences between the initial-short and initial-long condition, we speculate that this will be true for active conditions, especially at similar pennation angles compared to maximal passive plantarflexion. Furthermore, we assume that dynamic contractions can be used for the DIT method instead of using passive movements. One limitation of the current study is the low number of subjects used for the UFI condition. Inclusion of extra subjects would have increased the statistical power for these analyses, yet by using both legs, power was slightly increased, as both legs can be considered as independent measures due to the large bilateral differences in muscle fascicle architecture (Aeles et al., 2017).

\section{Conclusions and recommendations}

In conclusion, we have shown that DIT does not result in lower SEM values but a higher reliability between the raters was found, suggesting a more consistent fascicle identification. SEM values were lower however when using initial-short image sequences, yet this effect may be cancelled when calculating differences relatively to the absolute fascicle length. Overall, studies interested in single-image fascicle lengths are advised to record image sequences with fascicle length changes prior to or after the fascicle length of interest. For these image sequences, we have shown that the difference in fascicle length on the initial image explains most of the variability between the fascicle lengths of different raters. Differences between raters in fascicle lengths of image sequences are still relatively high, yet, when calculated relative to the fascicle length they appear independent of the fascicle length on the initial image and the results show high similarity and consistency. However, caution is needed when comparing fascicle lengths between different studies, mainly in terms of absolute values (e.g. total fascicle length changes). Studies that look at fascicle length changes over time, such as longitudinal training studies, should take these findings into account and should look to achieve high consistency between pre 
410 and post measurements and maximize the reliability between pre and post analyses. For example,

411 Aeles et al. (2017) suggested that muscle fascicle lengths should be measured at a muscle-tendon

412 unit length where there is no tension on the muscle-tendon unit and showed large variations

413 between subjects in the ankle joint angle at which this is true for the medial gastrocnemius. It

414 remains unknown whether this joint angle changes following a training intervention. As such,

415 these joint angles should be determined for each individual in both the pre and post

416 measurement. Additionally, by applying the DIT method and having the fascicle identification

417 done by a single rater, the reliability of the analysis can be increased. Furthermore, both the

418 intra-rater error as well as the inter-rater error should be taken into account when drawing

419 conclusions from comparisons of absolute fascicle lengths in both static and dynamic conditions

420 either within one study or between different studies. SEM from our raters ranged from 1.5 to $5 \%$

421 of absolute fascicle length within one rater and was around 7\% between different raters. These

422 values are not far from the typically reported effect sizes mentioned in the introduction and are

423 within the range of previously reported SEM values. Our analyses on relative differences

424 between raters in absolute fascicle length showed average values of $8.12-8.98 \%$. As such, we

425 recommend caution when drawing conclusions from fascicle length comparisons with

426 differences below these values of roughly $9 \%$ of absolute fascicle length. Combined, our

427 findings suggest that fascicle identification on ultrasound images is best done by one rater and

428 that the error due to the manual fascicle identification should be taken into account when

429 comparing absolute fascicle lengths between different raters or between different studies.

430 Finally, we urge that other strategies for improvement of the initial image fascicle identification

431 on ultrasound images should be studied. 


\section{References}

433 1. Abe T, Kumagai K, Brechue WF (2000) Fascicle length of leg muscles is greater in sprinters than distance runners. Med Sci Sports Exerc 32: 1125-1129

2. Aeles J, Lenchant S, Vanlommel L, Vanwanseele B (2017) Bilateral differences in muscle

3. Blazevich AJ (2006) Effects of physical training and detraining, immobilization, growth and

5. Brennan SF, Cresswell AG, Farris DJ, Lichtwark GA (2017) The effect of cadence on the muscle-tendon mechanics of the gastrocnemius muscle during walking. Scand J Med Sci Sports 27: $289-298$

6. Cronin NJ, Carty CP, Barrett RS, Lichtwark GA (2011) Automatic tracking of medial gastrocnemius fascicle length during human locomotion. J Appl Physiol 111: 1491-1496

7. Cronin NJ, Lichtwark GA (2013) The use of ultrasound to study muscle-tendon function in human posture and locomotion. Gait Posture 37: 305-312

8. Duclay J, Martin A, Duclay A, Cometti G, Pousson M (2009) Behavior of fascicles and the myotendinous junction of human medial gastrocnemius following eccentric strength training. Muscle Nerve 39: 819-827

9. Farris DJ, Sawicki GS (2012) Human medial gastrocnemius force-velocity behavior shifts with locomotion speed and gait. Proc Natl Acad Sci 109: 977-982

10. Farris DJ, Lichtwark GA (2016) Ultratrack: software for semi-automated tracking of muscle fascicles in sequences of B-mode ultrasound images. Comput Methods Programs Biomed 128: $111-118$ 
11. Farris DJ, Lichtwark GA, Brown NAT, Cresswell AG (2016) The role of human ankle plantar flexor muscle-tendon interaction and architecture in maximal vertical jumping examined in vivo. J Exp Biol 219: 528-534.

12. Farris DJ, Lichtwark GA, Brown NAT, Cresswell AG (2016) Deconstructing the power-

14. Franchi MV, Atherton PJ, Reeves ND, Flück M, Williams J, Mitchell WK, Selby A, Beltran Valls RM, Narici MV (2014) Architectural, functional and molecular responses to concentric and eccentric loading in human skeletal muscle. Acta Physiol (Oxf) 210: 642-654

15. Fukunaga T, Kawakami Y, Kuno S, Funato K, Fukashiro S (1997) Muscle architecture and function in humans. J Biomech 30: 457-463.

16. Fukunaga T, Kubo K, Kawakami Y, Fukashiro S, Kanehisa H, Maganaris CN (2001) In vivo behavior of human muscle tendon during walking. Proc R Soc Lond B 268: 229-233.

17. Fukutani A, Kurihara T (2015) Comparison of the muscle fascicle length between resistance-trained and untrained individuals: cross-sectional observation. SpringerPlus 4: 341

18. Gillet JG, Barrett RS, Lichtwark GA (2013) Reliability and accuracy of an automated tracking algorithm to measure controlled passive and active muscle fascicle length changes from ultrasound. Comput Methods Biomech Biomed Engin 16: 678-687

19. Herbert RD, Clarke J, Kwah LK, Diong J, Martin J, Clarke EC, Bilston LE, Gandevia SC (2011) In vivo passive mechanical behaviour of muscle fascicles and tendons in human gastrocnemius muscle-tendon units. J Physiol 21: 5257-5267

20. Hoffer JA, Caputi AA, Pose IE, Griffiths RI (1989) Roles of muscle activity and load on the relationship between muscle spindle length and whole muscle length in the freely walking cat. Prog Brain Res 80: 75-85 
484

485

486

487

488

489

490

491

492

493

494

495

496

497

498

499

500

501

502

503

504

505

506

507

21. Hoffman BW, Cresswell AG, Carroll TJ, Lichtwark GA (2016) Protection from muscle damage in the absence of changes in muscle mechanical behavior. Med Sci Sports Exerc 48: 1495-1505

22. Ishikawa M, Komi PV, Grey MJ, Lepola V, Bruggemann GP (2005) Muscle-tendon interaction and elastic energy usage in human walking. J Appl Physiol 99: 603-608

23. Ishikawa M, Pakaslahti J, Komi PV (2007) Medial gastrocnemius muscle behavior during human running and walking. Gait Posture 25: 380-384

24. Karamanidis K, Albracht K, Braunstein B, Catala MM, Goldmann JP, Brüggemann GP (2011)

Lower leg musculoskeletal geometry and sprint performance. Gait Posture 34: 138-141

25. König N, Cassel M, Intziegianni K, Mayer F (2014) Inter-rater reliability and measurement error of sonographic muscle architecture assessments. J Ultrasound Med 33: 769-777

26. Kurokawa S, Fukunaga T, Fukashiro S (2001) Behavior of fascicles and tendinous structures of human gastrocnemius during vertical jumping. J Appl Physiol 90: 1349-1358.

27. Kwah LK, Pint RZ, Diong J, Herbert RD (2013) Reliability and validity of ultrasound measurements of muscle fascicle length and pennation in humans: a systematic review. J Appl Physiol 114: 761-769

28. Lichtwark GA (2017) Ultrasound technology for examining the mechanics of the muscle, tendon, and ligament. In: Müller B, Wolf SI (ed) Handbook of Human Motion, $1^{\text {st }}$ edn. Springer International Publishing AG, Cham, pp 1-20

29. Lichtwark GA, Wilson AM (2006) Interactions between the human gastrocnemius muscle and the Achilles tendon during incline, level and decline locomotion. J Exp Biol 209: 4379-4388

30. Lichtwark GA, Wilson AM (2007) Muscle fascicle and series elastic element length changes along the length of the human gastrocnemius during walking and running. J Biomech 40: 157-164

31. Loram IA, Maganaris CN, Lakie M (2004) Paradoxical muscle movement in human standing. J Physiol 556: 683-689 
32. Magnusson SP, Hansen P, Aagaard P, Brønd J, Dyhre-Poulsen P, Bojsen-Moller J, Kjaer M (2003) Differential strain patterns of the human gastrocnemius aponeurosis and free tendon, in vivo. Acta Physiol Scand 177: 185-195

33. McMahon JJ, Turner A, Comfort P (2016) Within- and between-session reliability of medial gastrocnemius architectural properties. Biol Sport 33: 185-188

34. Mohagheghi AA, Khan T, Meadows TH, Giannikas K, Baltzopoulos V, Maganaris CN (2007) Differences in gastrocnemius muscle architecture between the paretic and non-paretic legs in children with hemiplegic cerebral palsy. Clin Biomech (Bristol, Avon) 22: 718-724

35. Rana M, Hamarneh G, Wakeling JM (2009) Automated tracking of muscle fascicle orientation in B-mode ultrasound images. J Biomech 42: 2068-2073

36. Sakuma J, Kanehisa H, Yanai T, Fukunaga T, Kawakami Y (2012) Fascicle-tendon behavior of the gastrocnemius and soleus muscles during ankle bending exercise at different movement frequencies. Eur J Appl Physiol 112: 887-898

37. Seiberl W, Hahn D, Kreuzpointner F, Schwirtz A, Gastmann U (2010) Force enhancement of quadriceps femoris in vivo and its dependence on stretch-induced muscle architectural changes. J Appl Biomech 26: 256-264

38. Seymore KD, Domire ZJ, DeVita P, Rider PM, Kulas AS (2017) The effect of Nordic hamstring strength training on muscle architecture, stiffness, and strength. Eur J Appl Physiol 117: 943-953

39. Theis N, Korff T, Kairon H, Mohagheghi AA (2013) Does acute passive stretching increase muscle length in children with cerebral palsy. Clin Biomech (Bristol, Avon) 28: 1061-1067

40. Timmins RG, Bourne MN, Shield AJ, Williams MD, Lorenzen C, Opar DA (2016) Biceps femoris architecture and strength in athletes with a previous anterior cruciate ligament reconstruction. Med Sci Sports Exerc 48: 337-345 


\section{Figure captions}

532

533 Fig. 1. Schematic summary of the data collection and further categorisation of the different

534 datasets for final analyses. DIT = data-informed tracking; UFI = uninformed fascicle

535 identification. B shows the comparison between initial-long and initial-short conditions for the

536 DIT condition. A shows the DIT and UFI comparison.

537

538 Fig. 2. Example set of three waveforms without (A) and with (B) the initial-frame offset

539 removed, one from each rater, for the initial-long condition. The black solid waveform is the

540 tracking from the reference rater. Data analyses methods and results are shown for (the

541 differences between) the grey dashed waveform and the black solid waveform as an example.

542 Note that the values in the results section are average differences between all three waveforms.

543

544 Fig. 3. Bland-Altman plot of the difference in total fascicle length change during the passive 545 rotation between rater $1\left(R_{1}\right)$ and the reference rater $\left(R_{\text {ref }}\right)$ (black $\left.{ }^{*}\right)$ and rater $2\left(R_{2}\right)$ and $R_{\text {ref }}($ grey $546 \diamond$ ) on the $y$-axis. X-axis shows the total length change values of $R_{\text {ref }}$ for the initial-long condition. 547 Mean differences between $\mathrm{R}_{1}$ and $\mathrm{R}_{\text {ref }}$ are shown by the black solid line and between $\mathrm{R}_{2}$ and $\mathrm{R}_{\text {ref }}$ 548 by the grey solid line. The black dashed lines give the upper and lower boundary of 1.96 times

549 the standard deviation (SD) for the difference between $\mathrm{R}_{1}$ and $\mathrm{R}_{\text {ref }}$, the grey dotted lines show 550 this for the difference between $R_{2}$ and $R_{\text {ref. }}$. 


\section{Figure 1 (on next page)}

\section{Protocol overview}

Graphical summary of the data collection and further categorisation of the different datasets for final analyses. DIT = data-informed tracking; UFI = uninformed fascicle identification. $\mathrm{B}$ shows the comparison between initial-long and initial-short conditions for the DIT condition. A shows the DIT and UFI comparison. 


$$
(\mathrm{N}=28)
$$

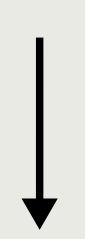

image sequences

$$
(\mathrm{N}=28)
$$

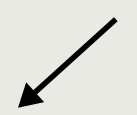

Left leg

1 recording

$(\mathrm{N}=28)$

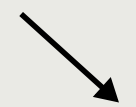

Right leg

1 recording

$(\mathrm{N}=28)$

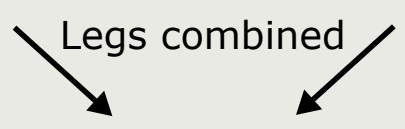

Each rotation split at maximum

$$
(\mathrm{N}=56)
$$
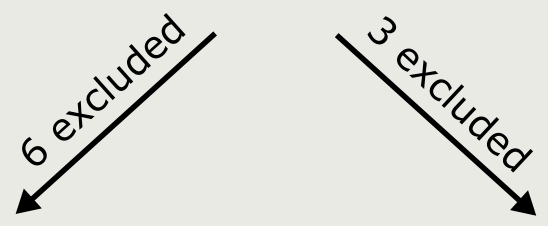

DIT

Initial-short

$(N=50)$
DIT

Initial-long

$$
(N=53)
$$

single-images

$(\mathrm{N}=5)$

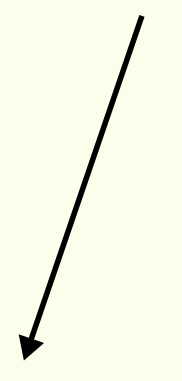

Left leg 3 images $(\mathrm{N}=15)$

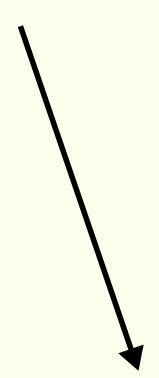

Right leg 3 images $(\mathrm{N}=15)$ 


\section{Figure 2}

Analyses example

Example set of three waveforms without $(A)$ and with $(B)$ the initial-frame offset removed, one from each rater, for the initial-long condition. The black solid waveform is the tracking from the reference rater. Data analyses methods and results are shown for (the differences between) the grey dashed waveform and the black solid waveform as an example. Note that the values in the results section are average differences between all three waveforms. 

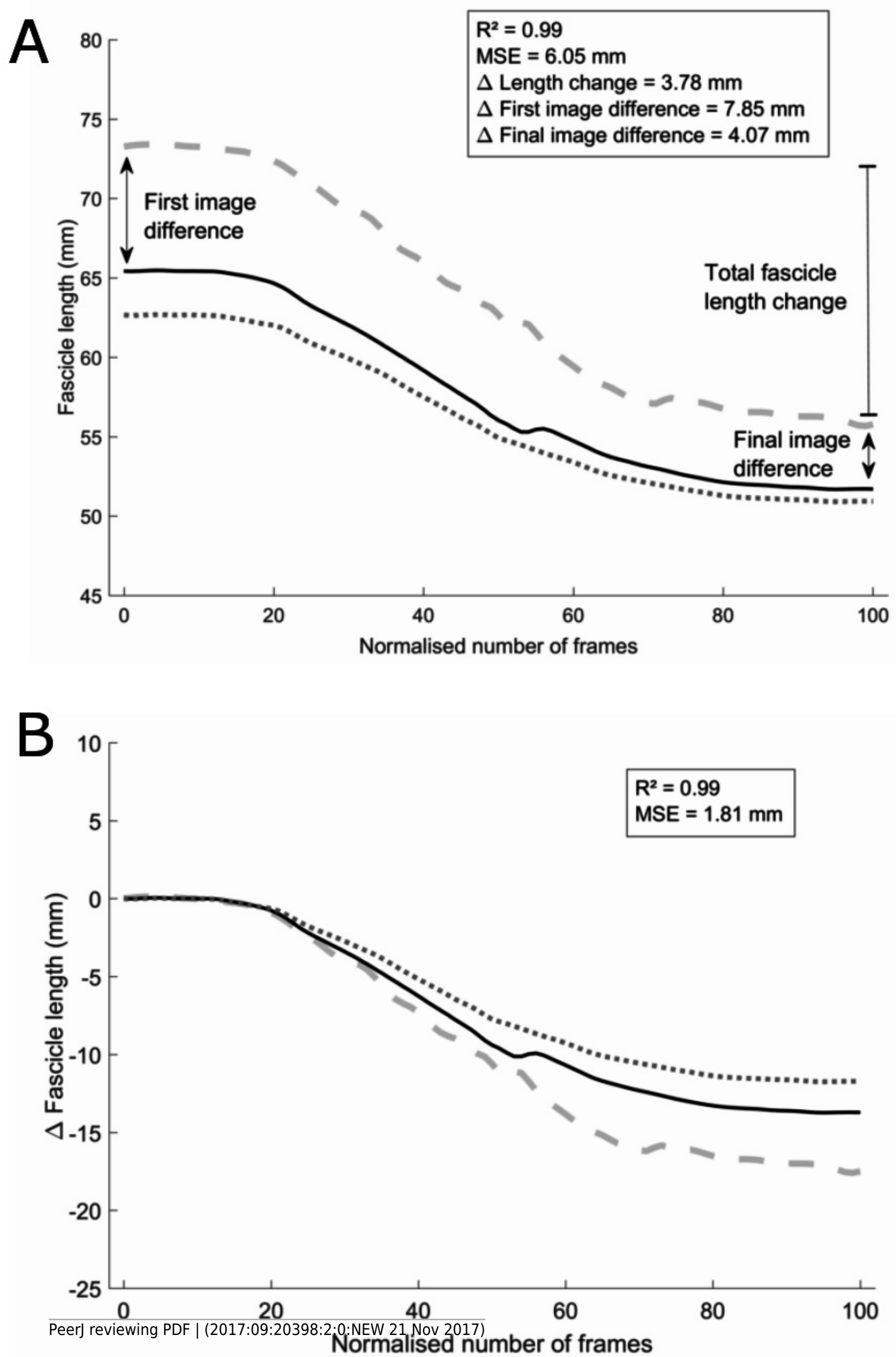


\section{Figure 3}

Bland-Altman plot

Bland-Altman plot of the difference in total fascicle length change during the passive rotation between rater $1\left(R_{1}\right)$ and the reference rater $\left(R_{\text {ref }}\right)$ (black $\left.*\right)$ and rater $2\left(R_{2}\right)$ and $R_{\text {ref }}$ (grey $\left.\diamond\right)$ on the $y$-axis. X-axis shows the total length change values of $R_{\text {ref }}$ for the initial-long condition. Mean differences between $R_{1}$ and $R_{\text {ref }}$ are shown by the black solid line and between $R_{2}$ and $R_{\text {ref }}$ by the grey solid line. The black dashed lines give the upper and lower boundary of 1.96 times the standard deviation (SD) for the difference between $R_{1}$ and $R_{\text {ref }}$, the grey dotted lines show this for the difference between $R_{2}$ and $R_{\text {ref. }}$.

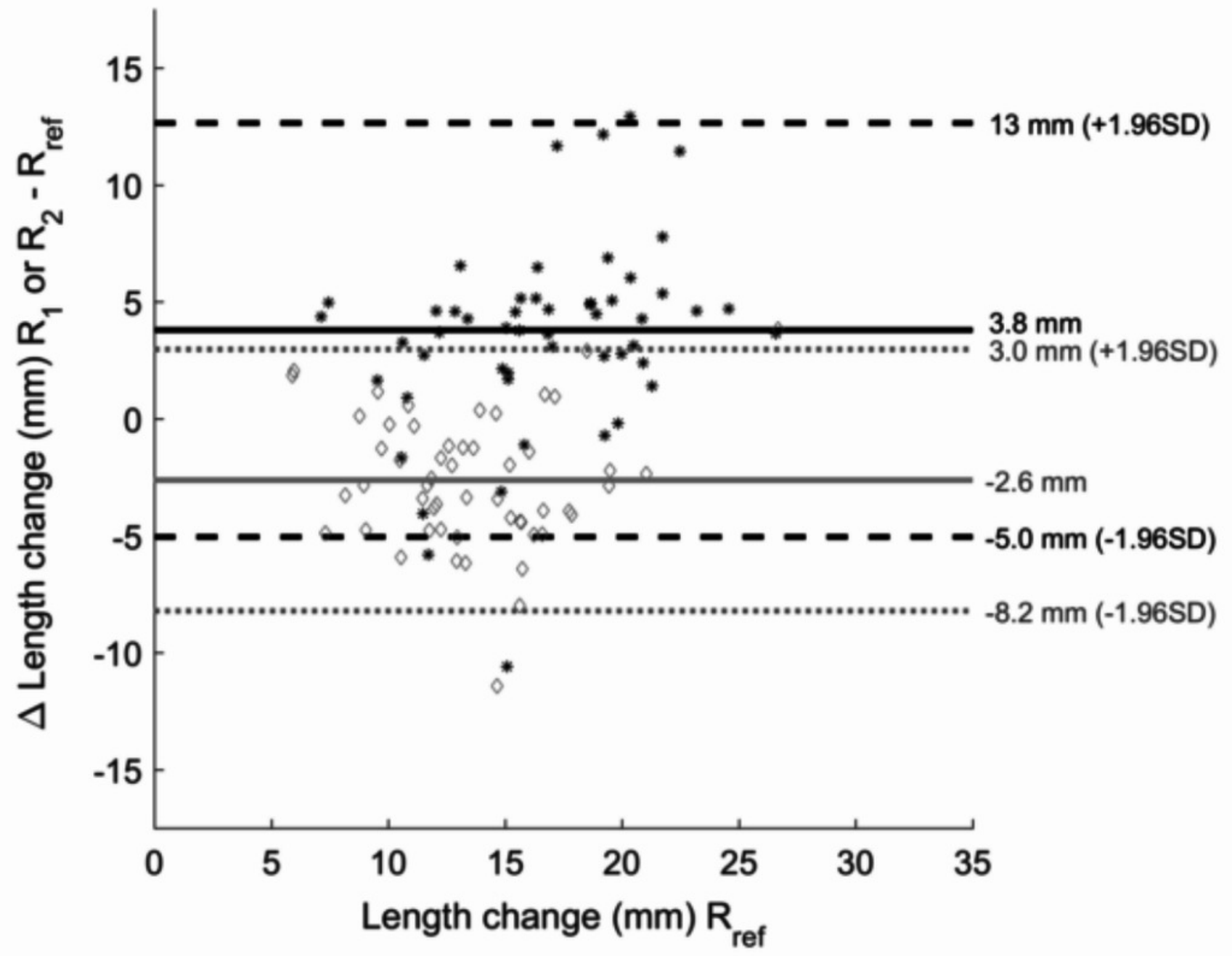




\section{Table $\mathbf{1}$ (on next page)}

DIT - UFI comparison results

Intra-class correlations and standard error of measurement values $(\mathrm{mm})$ for the datainformed tracking (DIT) and uninformed fascicle identification (UFI). 


\section{Tables}

2 Table 1. DIT - UFI comparison results

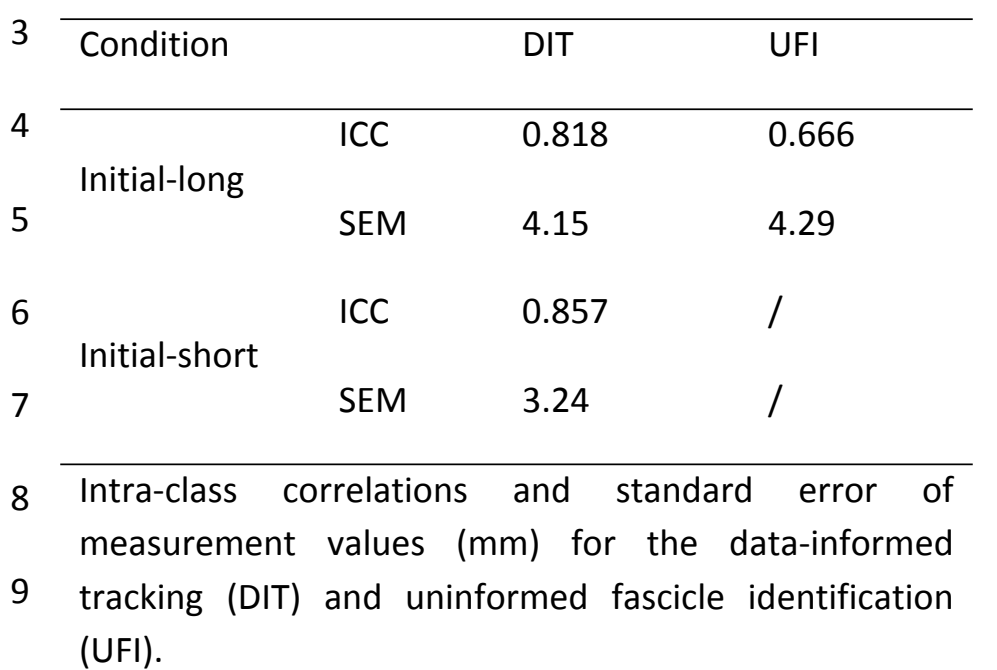




\section{Table 2 (on next page)}

CMD results for the initial-long and initial-short conditions

Values are means \pm SD. Coefficient of multiple determination (CMD) values approaching 1 denote high similarity of waveforms. Mean squared error (MSE) values are in $\mathrm{mm}$. * is significantly different compared to the offset-corrected condition $(p<0.05)$. 


\section{Tables}

Table 2. CMD results for the initial-long and initial-short conditions

\begin{tabular}{llll}
\hline Condition & $\mathrm{R}^{2}$ & $\mathrm{MSE}$ & MSE [offset-corrected] \\
\hline Initial-long & $0.98 \pm 0.05$ & $5.07 \pm 2.61^{*}$ & $2.75 \pm 1.38$ \\
Initial-short & $0.98 \pm 0.02$ & $5.30 \pm 2.36^{*}$ & $2.52 \pm 1.52$
\end{tabular}

2 Values are means \pm SD. Coefficient of multiple determination (CMD) values approaching 1 denote high 3 similarity of waveforms. Mean squared error (MSE) values are in $\mathrm{mm}$. ${ }^{*}$ is significantly different 4 compared to the offset-corrected condition $(p<0.05)$.

5

6 


\section{Table 3(on next page)}

Absolute differences between raters for physiologically-relevant parameters for the initial-long and initial-short conditions

Values are means $\pm S D$. All values represent the absolute differences between values of the different raters for that respective parameter. Fascicle length differences are in $\mathrm{mm}^{.}{ }^{\$}=$ significantly different from the initial-short condition. 
Tables

Table 3. Absolute differences between raters for physiologically-relevant parameters for the initial-long and initial-short conditions

\begin{tabular}{llll}
\hline Condition & $\begin{array}{l}\text { Total fascicle } \\
\text { length change }\end{array}$ & $\begin{array}{l}\text { Fascicle length - } \\
\text { first image }\end{array}$ & $\begin{array}{l}\text { Fascicle length - } \\
\text { final image }\end{array}$ \\
\hline Initial-long & $4.83 \pm 2.56$ & $6.14 \pm 3.16^{\$}$ & $4.42 \pm 2.58^{\$}$ \\
Initial-short & $3.97 \pm 2.80$ & $4.54 \pm 2.43$ & $6.15 \pm 3.11$ \\
\hline
\end{tabular}

Values are means \pm SD. All values represent the absolute differences between values of the different raters for that respective parameter. Fascicle length differences are in $\mathrm{mm} .{ }^{\$}=$ significantly different from the initial-short condition. 


\section{Table 4 (on next page)}

Relative differences between raters for physiologically-relevant parameters for the initial-long and initial-short conditions

Values are means $\pm S D$. All values represent the relative differences between values of the different raters for that respective parameter in $\%$ of the absolute total fascicle length change and absolute fascicle length on the first and final image respectively. 
Table 4. Relative differences between raters for physiologically-relevant parameters for the initial-long and initial-short conditions

\begin{tabular}{llll}
\hline Condition & $\begin{array}{l}\text { Total fascicle } \\
\text { length change }\end{array}$ & $\begin{array}{l}\text { Fascicle length - } \\
\text { first image }\end{array}$ & $\begin{array}{l}\text { Fascicle length - } \\
\text { final image }\end{array}$ \\
\hline Initial-long & $34.29 \pm 17.96$ & $8.98 \pm 5.12$ & $8.34 \pm 4.97$ \\
Initial-short & $31.01 \pm 18.86$ & $8.12 \pm 4.29$ & $8.89 \pm 4.55$ \\
\hline
\end{tabular}

Values are means \pm SD. All values represent the relative differences between values of the different raters for that respective parameter in \% of the absolute total fascicle length change and absolute fascicle length on the first and final image respectively. 